also

$$
\bar{\gamma}=-\alpha^{q_{1}} \alpha^{q_{2}} .
$$

In (63) eingesetzt:

$$
\begin{gathered}
\bar{\lambda}_{p_{1} p_{\mathrm{g}}=} \alpha_{p_{1}} \alpha_{p_{\mathrm{s}}} \alpha^{q_{1}} \alpha^{q_{\mathrm{s}}} D^{-1}(\varepsilon+i o) \\
\left(p_{10}+p_{20}-\varepsilon-i o\right)^{-1} .
\end{gathered}
$$

In der Tat ist dann wegen (62) die Gl. (69) erfüllt. Damit ist $\lambda$ völlig bestimmt. Es folgt :

1 T. D. LeE, Phys. Rev. 95, 1329 [1954].

2 G. Källén u. W. Pauli, Kgl. Danske Vidensk. Selskab, Mat.Fys. Medd. 30, 7 [1955].

3 R. D. Amado u. R. P. Kenschaft, J. Math. Phys. 5, 1340 [1964].

4 A. Pagnamenta, J. Math. Phys. 6, 955 [1965] ; 7, 356 [1966].

5 C. M. Sommerfield, J. Math. Phys. 6, 1170 [1965].

$$
\begin{gathered}
\psi^{q_{1} q_{2}}=\chi^{q_{1} q_{2}}+\alpha^{q_{1}} \alpha^{q_{2}} D^{-1}\left(q_{10}+q_{20}+i o\right) \\
\int \mathrm{d} p_{1} \mathrm{~d} p_{2} \alpha_{p_{1}} \alpha_{p_{2}} \chi^{p_{1} p_{2}}\left(p_{10}+p_{20}-q_{10}-q_{20}-i o\right)^{-1} .
\end{gathered}
$$

Wie in ${ }^{12}$ gezeigt wird, ist $\psi^{q_{1} q_{2}}$ tatsächlich eine Lösung der Schrödinger-Gleichung. Im Falle eines instabilen $V$-Teilchens stellen die $\psi^{q_{1} q_{2}}$ ein vollständiges orthonormiertes System im $N \Theta \Theta$-Sektor dar.

6 E. Kazes, J. Math. Phys. 6, 1172 [1965].

7 J. C. Houard, Ann. Inst. Henri Poincaré A II, 105 [1965].

8 G. L. Tindle, Nuovo Cim. A 45, 619 [1966].

9 D. I. Fivel, J. Math. Phys. 11, 699 [1970].

10 M. Bolsterli, Phys. Rev. 166, 1760 [1968].

11 H. v. Dewitz u. K. Helmers, Z. Naturforsch. 26 a, 4 [1971].

12 H. v. Dewitz, Diplomarbeit.

\title{
The Energy-Density Functional of an Electron Gas in Locally Linear Approximation of the One-Body Potential *
}

\author{
R. BALTIN \\ Institut A für Theoretische Physik der Technischen Universität Braunschweig, Germany \\ (Z. Naturforsch. 27 a, 1176-1186 [1972] ; received 11 March 1972)
}

\begin{abstract}
For a system of independent electrons moving in a common one-body potential $V(\boldsymbol{r})$ an integral representation of Dirac's density matrix is evaluated in the approximation that $V(\boldsymbol{r})$ at the point $\boldsymbol{r}$ is replaced by a linear potential with a gradient equal to the gradient of $V$ at $\boldsymbol{r}$. The particle density $\varrho, \nabla \varrho$ and the kinetic-energy density $\varepsilon_{\mathrm{k}}$ are derived from the density matrix. After eliminating the potential and its gradient a parametric representation for $\varepsilon_{\mathrm{k}}$ in terms of $\varrho$ and $y \equiv|\nabla \varrho|^{1 / 2} \varrho^{-2 / 3}$ is obtained. Explicit analytical expressions are given in the limits $y \rightarrow 0$ and $y \rightarrow \infty$ and compared with the inhomogeneity corrections of Kirzhnits and v. Weizsäcker.
\end{abstract}

\section{Introduction}

According to the original Thomas-Fermi theory the kinetic-energy density $\varepsilon_{\mathrm{k}}$ of electrons with particle density $\varrho$ moving independently in a common effective one-body potential $V(\boldsymbol{r})$ is given by

$$
\varepsilon_{\mathrm{k}}=\varkappa \varrho^{5 / 3}\left[\varkappa=0.3\left(3 \pi^{2}\right)^{2 / 3} \hbar^{2} / m\right] .
$$

As is well-known, this relation is an insufficient approximation either when the potential varies rapidly in space or when the density is very low. For the general case of an inhomogeneous electron gas, HoHENBERG and KOHN ${ }^{1}$ have shown that the ground state kinetic energy is a unique functional of $\varrho$ whether or not the electrons are interacting. However, it seems difficult to gain valid explicit approximations to this functional beyond the TF-term.

Reprints request to Dr. R. BALTin, Institut A für Theoretische Physik, Technische Universität, D-3300 Braunschweig, Germany.

* Extracted from the author's doctoral thesis (D 84).
In a rather intuitive approach, v. WEIZsÄCKER ${ }^{2}$ introduced an additive correction term $\sim(\nabla \varrho)^{2} / \varrho$ to $\varepsilon_{\mathrm{k}}^{\mathrm{TF}}$ whereby qualitative improvements could be achieved. However, application to some specific potentials yields too high ground state energies ${ }^{3-5}$.

A systematic investigation of KIRZHNITs ${ }^{6}$ starting from a reformulation of the Hartree-Fock equations in terms of Dirac's density matrix and expanding the latter in a power series of $\hbar$ came to the result that for weak inhomogeneity the Weizsäcker correction term should be multiplied by a factor of $\gamma=1 / 9$. Other authors ${ }^{7-9}$ using similar methods obtain the same factor. Golden ${ }^{10}$ finds $\gamma=13 / 45$. Using semiclassical arguments GомвÁs ${ }^{11-13}$ claims that not the Weizsäcker term but the TF term should be corrected by a factor dependent upon the number of electrons.

Several authors ${ }^{14-16}$ have pointed out that Kirzhnits' series expansion doesn't take into account the nonanalytical behaviour of Dirac's density matrix 
with respect to $\hbar$. Consequently, quantum oscillations are missing in that treatment. Furthermore, the Kirzhnits series appears to be merely asymptotic.

Concerning applications of the Kirzhnits correction, calculations of total energies of atoms ${ }^{17}$ and other atomic properties ${ }^{18}$ lead to rather disappointing results. Recently, JONES and YouNG ${ }^{19}$ showed that in the case of a weakly perturbed extended system of noninteracting fermions the correct ground state energy is obtained if for very small perturbation wavelength the full Weizsäcker functional and for very large wavelength the Kirzhnits functional is used.

Starting from a free electron gas, STODDART and MARCH ${ }^{20}$ have established a functional by perturbation theory to infinite order. Unfortunately, their expression is difficult to evaluate.

In the present paper, the author has treated this problem from a point of view somewhat different from previous work on this subject.

In deriving $\varepsilon_{\mathrm{k}}^{\mathrm{TF}}$ it has been assumed that the whole space may be divided into volume cells each of which containing many electrons, but being yet so small that the potential inside a single cell can be replaced by a mean value $V_{0}$. In this approximation the electrons in each cell are free and therefore occupy the planewave states of a local Fermi sphere whence the above expression for $\varepsilon_{\mathrm{k}}^{\mathrm{TF}}$ is obtained after $V_{0}$ has been eliminated. As next approximation, it seems reasonable now to replace the potential in the cell by a linear one which has the same value and the same gradient as $V$ at some point $\boldsymbol{r}_{0}$ within the cell. This approximation which has already been used by SWIATECKI ${ }^{21}$ for calculation of $\varrho$ shall be adopted here in order to derive the energy-density functional. No further approximation shall be made. The plan of the work is as follows:

In Sect. 2 an integral representation of Dirac's density matrix $\varrho\left(\boldsymbol{r}, \boldsymbol{r}^{\prime}\right)$ is introduced which is evaluated in Sect. 3 after using the approximation described. In Sect. $4 \varrho$ and $\nabla \varrho$ are calculated from $\varrho\left(\boldsymbol{r}, \boldsymbol{r}^{\prime}\right)$ as functions of $\lambda-V(\boldsymbol{r})$ and $\nabla V(\boldsymbol{r})$ where $\lambda$ is a constant necessary for normalizing $\varrho$. In Sect. 5 from $\varrho\left(\boldsymbol{r}, \boldsymbol{r}^{\prime}\right)$ the kinetic-energy density is obtained depending likewise upon $\lambda-V$ and $\nabla V$. Eliminating the latter quantities by means of $\varrho$ and $\nabla \varrho$ we arrive at a parametric representation of $\varepsilon_{\mathbf{k}}(\varrho, \nabla \varrho)$ which is evaluated numerically for general values in Section 6. In the limits $\varrho^{-2 / 3}|\nabla \varrho|^{1 / 2} \ll 1$ and $\gg 1$ explicit analytical expressions of $\varepsilon_{\mathrm{k}}$ can be given
(Sect. 7). In the last section the results are discussed.

\section{Dirac's Density Matrix in Integral Representation}

Let us consider the ground state of an electron gas in Hartree-approximation where each electron (position vector $\boldsymbol{r}$ ) is assumed to move independently in a selfconsistent one-body potential $\mathrm{V}(\boldsymbol{r})$. Then the state function of the whole system is a Slater determinant made up of one-electron wave functions obeying the Schrödinger equation

$$
\hat{\boldsymbol{H}} \psi_{n, \mu}(\boldsymbol{r})=\varepsilon_{n} \psi_{n, \mu}(\boldsymbol{r}), \quad n=1,2, \ldots
$$

with

$$
\hat{H}=-\frac{\hbar^{2}}{2 m} \Delta \boldsymbol{r}+V(\boldsymbol{r}) .
$$

For simplicity, the spectrum is assumed to be discrete, so the energy levels may be labelled in ascending order, i. e., $\varepsilon_{1}<\varepsilon_{2}<\varepsilon_{3} \ldots$ The index $\mu$ allows for distinction between wave functions belonging to a degenerate level. The $\psi_{n, \mu}$ 's are supposed to satis fy the conditions of orthonormality and completeness. Let the level $\varepsilon_{n}$ be $m(n)$-fold degenerate. Then Dirac's density matrix is defined as

$$
\varrho\left(\boldsymbol{r}, \boldsymbol{r}^{\prime}\right)=2 \sum_{n^{\prime}=1}^{n} \sum_{\mu^{\prime}=1}^{m\left(n^{\prime}\right)} \psi_{n^{\prime}, \mu^{\prime}}^{*}\left(\boldsymbol{r}^{\prime}\right) \psi_{n^{\prime}, \mu^{\prime}}(\boldsymbol{r}) .
$$

In the ground state the electron density is given by $\varrho(\boldsymbol{r}, \boldsymbol{r})$ when all levels are occupied up to $\varepsilon_{n}$. Following the work of ALFRED ${ }^{22}$ and MACKE and RENNERT ${ }^{23}$ we now use an integral representation of $\varrho\left(\boldsymbol{r}, \boldsymbol{r}^{\prime}\right)$ where the individual wave functions are eliminated. By means of the step function $\theta(u)$ we can write

$\varrho\left(\boldsymbol{r}, \boldsymbol{r}^{\prime}\right)=2 \sum_{n^{\prime}=1}^{\infty} \sum_{\mu^{\prime}=1}^{m\left(n^{\prime}\right)} \boldsymbol{\theta}\left(\lambda-\varepsilon_{n^{\prime}}\right) \psi_{n^{\prime}, \mu^{\prime}}^{*}\left(\boldsymbol{r}^{\prime}\right) \psi_{n^{\prime}, \mu^{\prime}}(\boldsymbol{r})$

where the parameter $\lambda$ satisfies the inequality

$$
\varepsilon_{n}<\lambda<\varepsilon_{n+1} \text {. }
$$

Since the $\psi_{n, \mu}$ are eigenfunctions of the real operator $\hat{H}$ we may write

$$
\begin{aligned}
\boldsymbol{\theta}\left(\lambda-\varepsilon_{n}\right) \psi_{n, \mu}^{*}\left(\boldsymbol{r}^{\prime}\right) \psi_{n, \mu}(\boldsymbol{r}) & (2.6) \\
= & \theta\left[\lambda-\frac{1}{2}\left(\hat{H}+\hat{H}^{\prime}\right)\right] \psi_{n, \mu}^{*}\left(\boldsymbol{r}^{\prime}\right) \psi_{n, \mu}(\boldsymbol{r})
\end{aligned}
$$

where $\hat{H}^{\prime}$ acts upon functions of $\boldsymbol{r}^{\prime}$. 
Inserting this expression which is symmetric with respect to interchange of $\boldsymbol{r}$ and $\boldsymbol{r}^{\prime}$ into Eq. (2.4) we are able to take the operator function out of the summation signs and to apply the closure condition

$$
\varrho\left(\boldsymbol{r}, \boldsymbol{r}^{\prime}\right)=2 \theta\left[\lambda-\frac{1}{2}\left(\hat{H}+\hat{H}^{\prime}\right)\right] \delta\left(\boldsymbol{r}-\boldsymbol{r}^{\prime}\right) .
$$

Using the well-known representations for $\theta(u)$ and for the $\delta$-function

$$
\theta(u)=\frac{1}{2 \pi i} \int_{-\infty-i \eta}^{\infty-i \eta} \frac{\mathrm{d} \omega}{\omega} e^{i \omega u},
$$

$\eta$ being a positive infinitesimal, and

$$
\delta\left(\boldsymbol{r}-\boldsymbol{r}^{\prime}\right)=\frac{1}{(2 \pi)^{3}} \int_{-\infty}^{+\infty} \mathrm{d}^{3} \boldsymbol{k} \cdot \exp \left\{-i \boldsymbol{k}\left(\boldsymbol{r}-\boldsymbol{r}^{\prime}\right)\right\}
$$

we obtain

$$
\begin{aligned}
& \varrho\left(\boldsymbol{r}, \boldsymbol{r}^{\prime}\right)=\frac{1}{i \pi(2 \pi)^{3}} \int_{-i \eta}^{+\infty} \frac{\mathrm{d} \omega}{\omega} \int_{-\infty}^{+\infty} \mathrm{d}^{3} \boldsymbol{k} \\
& \cdot \exp \left\{i \omega\left(\lambda-\frac{1}{2}\left[\hat{H}+\hat{H}^{\prime}\right]\right)\right\} \exp \left\{i \boldsymbol{k}\left(\boldsymbol{r}^{\prime}-\boldsymbol{r}\right)\right\} .
\end{aligned}
$$

So we have shifted the problem of calculating and summing wave functions to the problem of evaluating and integrating an exponential operator acting upon a plane wave.

\section{Density Matrix in locally linear Approximation of the Potential}

For general $V(\boldsymbol{r})$ expression (2.10) can be evaluated only approximately because the kinetic energy operator $\hat{T}=(1 / 2 m) \hat{p}^{2}$ and the potential do not commute. To proceed further we shall make an aproximation to $V(\boldsymbol{r})$. Let us start with a Taylor series expansion of $V(\boldsymbol{r})$ about a fixed point

$$
\boldsymbol{r}_{0}=\left(x_{1}{ }^{(0)}, x_{2}{ }^{(0)}, x_{3}{ }^{(0)}\right)
$$

and break off after the gradient term:

with

$$
V(\boldsymbol{r}) \approx \bar{V}\left(\boldsymbol{r}_{0}\right)+\boldsymbol{r} \cdot \nabla V\left(\boldsymbol{r}_{0}\right)
$$

$$
\bar{V}\left(\boldsymbol{r}_{\mathbf{0}}\right)=V\left(\boldsymbol{r}_{\mathbf{0}}\right)-\boldsymbol{r}_{0} \cdot \nabla V\left(\boldsymbol{r}_{0}\right) .
$$

Noting $\left[\hat{p}_{i}, x_{j}^{\prime}\right]=\left[\hat{p}_{i}{ }^{\prime}, x_{j}\right]=0$ we can factorize the exponential in expression (2.10)

$$
\begin{aligned}
\exp \left\{i \omega \left(\lambda-\frac{1}{2}\right.\right. & \left.\left.\left(\hat{H}+\hat{H}^{\prime}\right)\right)\right\} \\
& =\exp \left\{i \omega\left(\lambda-\frac{1}{2}\left[\bar{V}\left(\boldsymbol{r}_{0}\right)+\bar{V}\left(\boldsymbol{r}_{0}{ }^{\prime}\right)\right]\right)\right\} \\
& \cdot \prod_{i=1}^{3}\left\{\hat{G}\left(\omega, \hat{p}_{i}, x_{i}\right) \hat{G}\left(\omega, \hat{p}_{i}{ }^{\prime}, x_{i}{ }^{\prime}\right)\right\}
\end{aligned}
$$

where

$$
\hat{G}\left(\omega, \hat{p}_{i}, x_{i}\right)=\exp \left\{-\frac{i \omega}{2}\left(\frac{\hat{p}_{i}{ }^{2}}{2 m}+\left.x_{i} \frac{\partial V}{\partial x_{i}}\right|_{\boldsymbol{r}=\boldsymbol{r}_{0}}\right)\right\} .
$$

$\hat{G}\left(\omega, \hat{p}_{i}, x_{i}\right)$ behaves as a $c$-number when acting upon a function which does not depend on $x_{i}$. Therefore we can write

$$
\begin{array}{r}
\exp \left\{i \omega\left(\lambda-\frac{1}{2}\left(\hat{H}+\hat{H}^{\prime}\right)\right)\right\} \exp \left\{i \boldsymbol{k}\left(\boldsymbol{r}^{\prime}-\boldsymbol{r}\right)\right\} \\
\quad=\exp \left\{i \omega\left(\lambda-\frac{1}{2}\left[\bar{V}\left(\boldsymbol{r}_{0}\right)+\bar{V}\left(\boldsymbol{r}_{0}^{\prime}\right)\right]\right)\right\} \cdot D_{1} D_{2} D_{3}
\end{array}
$$

with

$$
\begin{aligned}
D_{i}=\left[\hat{G}\left(\omega, \hat{p}_{i}, x_{i}\right) \exp \left\{-i k_{i} x_{i}\right\}\right] \\
\cdot\left[\hat{G}\left(\omega, \hat{p}_{i}^{\prime}, x_{i}^{\prime}\right) \exp \left\{i k_{i} x_{i}^{\prime}\right\}\right] .
\end{aligned}
$$

Since $D_{i}$ is independent of $k_{j}$ for $j \neq i$ we are able to decompose in Eq. (2.10) the three-dimensional integral over $\boldsymbol{k}$-space into a threefold product of onedimensional integrals over $k_{i}$ :

$$
\begin{aligned}
\varrho\left(\boldsymbol{r}, \boldsymbol{r}^{\prime}\right)= & \frac{1}{\pi i(2 \pi)^{3}} \int_{-\infty-i \eta}^{+\infty} \frac{\mathrm{d} \omega}{\omega} \\
& \cdot \exp \left\{i \omega\left(\lambda-\frac{1}{2}\left[\bar{V}\left(\boldsymbol{r}_{0}\right)+\bar{V}\left(\boldsymbol{r}_{0}{ }^{\prime}\right)\right]\right)\right\} \\
& \cdot \prod_{i=1}^{3} \int_{-\infty}^{+\infty} \mathrm{d} k_{i} D_{i}\left(\omega, x_{i}, x_{i}{ }^{\prime}, k_{i}\right) .
\end{aligned}
$$

The function $D_{i}$ is a product of factors of the form $\exp \left\{a \hat{p}^{2}+b x\right\} \cdot \exp \{i k x\}$, where $[\hat{p}, x]=-i \hbar$, and $a, b, k$ are $c$-numbers. There are various methods for calculating expressions of this kind ${ }^{15,23,24}$ the result being

$$
\begin{gathered}
\exp \left\{a \hat{p}^{2}+b x\right\} \cdot \exp \{i k x\}=\exp \left\{a \hbar^{2} k^{2}+b x\right. \\
\left.-i \hbar k a b-\frac{1}{3} \hbar^{2} b^{2} a\right\} \cdot \exp \{i k x\} .
\end{gathered}
$$

Using this relation we obtain from Eq. (3.4)

$$
D_{i}=A_{i} \cdot B_{i}
$$

where

$$
\begin{gathered}
A_{i}=\exp \left\{-i \beta k_{i}{ }^{2}+i \gamma_{i} k_{i}\right\}, \\
B_{i}=\exp \left\{-\frac{i \omega}{2}\left(\left.x_{i} \frac{\partial V}{\partial x_{i}}\right|_{r_{0}}+\left.x_{i}^{\prime} \frac{\partial V}{\partial x_{i}^{\prime}}\right|_{r_{0}}\right)\right. \\
\left.-\frac{i \hbar^{2} \omega^{3}}{48 m}\left[\left(\left.\frac{\partial V}{\partial x_{i}}\right|_{r_{0}}\right)^{2}+\left(\left.\frac{\partial V}{\partial x_{i}{ }^{\prime}}\right|_{r_{0^{\prime}}}\right)^{2}\right]\right\} \\
\beta=\hbar^{2} \omega / 2 m, \\
\gamma_{i}=x_{i}^{\prime}-x_{i}+\frac{\hbar^{2} \omega^{2}}{8 m}\left\{\left.\frac{\partial V}{\partial x_{i}^{\prime}}\right|_{\boldsymbol{r}_{0}^{\prime}}-\left.\frac{\partial V}{\partial x_{i}}\right|_{r_{0}}\right\} .
\end{gathered}
$$

Since $B_{i}$ does not depend upon $k_{i}$ we get

$$
\begin{aligned}
\int_{-\infty}^{+\infty} D_{i} \mathrm{~d} k_{i}= & B_{i} \int_{-\infty}^{+\infty} \exp \left\{-i \beta k^{2}+i \gamma_{i} k\right) \mathrm{d} k \\
& =B_{i} \cdot(i-1)\left(\frac{\pi}{2 \beta}\right)^{1 / 2} \exp \left\{\frac{i \gamma_{i}^{2}}{4 \beta}\right\} .
\end{aligned}
$$


Inserting this into Eq. (3.5) we find

$$
\begin{aligned}
\varrho\left(\boldsymbol{r}, \boldsymbol{r}^{\prime}\right)= & \frac{(i-1)^{3}}{8 \pi^{4} i} \int_{-\infty-i \eta}^{+\infty-i \eta} \frac{\mathrm{d} \omega}{\omega} \\
& \cdot \exp \left\{i \omega\left(\lambda-\frac{1}{2}\left[\bar{V}\left(\boldsymbol{r}_{0}\right)+\bar{V}\left(\boldsymbol{r}_{0}{ }^{\prime}\right)\right]\right)\right\} \\
& \cdot\left(\frac{\pi}{2 \beta}\right)^{3 / 2} B_{1} B_{2} B_{3} \exp \left\{\frac{i}{4 \beta}\left(\gamma_{1}{ }^{2}+\gamma_{2}{ }^{2}+\gamma_{3}{ }^{2}\right)\right\}
\end{aligned}
$$

We now substitute expressions $(3.8 \mathrm{~b}, \mathrm{c}, \mathrm{d})$ for $B_{i}$, $\beta$, and $\gamma_{i}$, and then replace $\nabla V\left(\boldsymbol{r}_{0}\right)$ by $\nabla V(\boldsymbol{r})$ and $\nabla V\left(\boldsymbol{r}_{0}{ }^{\prime}\right)$ by $\nabla V\left(\boldsymbol{r}^{\prime}\right)$ which amounts to neglecting higher derivatives of $V$ corresponding to the approximation we have made in Equation (3.1). After some algebra we find

$$
\varrho\left(\boldsymbol{r}, \boldsymbol{r}^{\prime}\right)=C \int_{-\infty-i \eta}^{+\infty} \frac{\mathrm{d} \omega}{\omega^{5 / 2}} \exp \left\{i \omega d_{1}+\frac{i d_{2}}{\omega}+i \omega^{3} d_{3}\right\}
$$

with

$$
\begin{gathered}
C=(1-i) m^{3 / 2} / 4 \pi^{5 / 2} \hbar^{3}, \\
d_{1}=\lambda-\frac{1}{2}\left[V(\boldsymbol{r})+V\left(\boldsymbol{r}^{\prime}\right)\right]+\frac{1}{8}\left(\boldsymbol{r}-\boldsymbol{r}^{\prime}\right) \\
\cdot\left(\left.\nabla V\right|_{r}-\left.\nabla V\right|_{r^{\prime}}\right), \\
d_{2}=m\left(\boldsymbol{r}-\boldsymbol{r}^{\prime}\right)^{2} / 2 \hbar^{2}, \\
d_{3}=\frac{\hbar^{2}}{16 m}\left\{\frac{1}{8}\left(\left.\nabla V\right|_{r}-\left.\nabla V\right|_{r^{\prime}}\right)^{2}\right. \\
-\frac{1}{3}\left[\left(\left.\nabla V\right|_{r} ^{2}+\left(\left.\nabla V\right|_{r^{\prime}}\right)^{2}\right]\right\}
\end{gathered}
$$

where Eq. (3.2) has been used. The density matrix does not contain $\boldsymbol{r}_{0}$ and $\boldsymbol{r}_{0}{ }^{\prime}$ any longer, and the position vectors $\boldsymbol{r}$ and $\boldsymbol{r}^{\prime}$ may vary unrestrictedly and independently from each other. Note that we have used the locally linear approximation to the given potential only to evaluate the integrand in Eq. (2.10), but afterwards, again the original potential has to be taken in the above expression for $\varrho\left(\boldsymbol{r}, \boldsymbol{r}^{\prime}\right)$.

\section{The Electron Density and Density Gradient}

From Eqs. (3.10), (3.11), and (3.12 a, b, c) we immediately obtain the electron density setting $\boldsymbol{r}=\boldsymbol{r}^{\prime}:$

$$
\begin{aligned}
& \varrho(\boldsymbol{r})=\varrho(\boldsymbol{r}, \boldsymbol{r})= C \int_{-\infty \eta}^{+\infty} \frac{\mathrm{d} \omega}{\omega^{5 / 2}} \\
& \cdot \exp \left\{i \omega(\lambda-V(\boldsymbol{r}))-\frac{i \omega^{3} \hbar^{2}}{24 m}|\nabla V|^{2}\right\} .
\end{aligned}
$$

As we shall see below $\varrho(\boldsymbol{r})$ is always nonnegative.

To remain consistent with our approximation, we have to keep $\nabla V(\boldsymbol{r})$ constant when calculating the gradient of $\varrho(\boldsymbol{r})$ because higher derivatives of $V$ than the first have been already neglected in gaining $\varrho$. Thus

$$
\begin{aligned}
\nabla \varrho(\boldsymbol{r})= & -i C \nabla V(\boldsymbol{r}) \int_{-\infty-i \eta}^{+\infty-i \eta} \frac{\mathrm{d} \omega}{\omega^{3 / 2}} \\
& \cdot \exp \left\{i \omega(\lambda-V(\boldsymbol{r}))-\frac{i \omega^{3} \hbar^{2}}{24 m}|\nabla V|^{2}\right\} .
\end{aligned}
$$

Defining

$$
\begin{gathered}
\xi=\lambda-V(\boldsymbol{r}), \\
\zeta=\left(\frac{\hbar^{2}|\nabla V|^{2}}{24 m}\right)^{1 / 3} \geqq 0, \\
R_{\nu}(z)=\int_{-\infty-i \eta}^{+\infty-i \eta} \frac{\mathrm{d} \tau}{\tau^{1+\nu}} \exp \left\{i \tau z-i \tau^{3}\right\},
\end{gathered}
$$

we may rewrite

$$
\begin{aligned}
\varrho(\boldsymbol{r}) & =C \zeta^{3 / 2} R^{3 / 2}(\xi / \zeta), \\
\nabla \varrho(\boldsymbol{r}) & =-i C \nabla V(\boldsymbol{r}) \zeta^{1 / 2} R_{1 / 2}(\xi / \zeta) .
\end{aligned}
$$

In order to improve convergence we introduce into Eq. (4.5) the identity

$$
e^{-i \tau^{3}}=3^{-1 / 3} \int_{-\infty}^{+\infty} \operatorname{Ai}\left(3^{-1 / 3} t\right) e^{i \tau t} \mathrm{~d} t
$$

where $\operatorname{Ai}(-x)$ denotes Airy's function. Reversing the order of integration and using the relation

$$
\begin{aligned}
& \int_{-\infty-i \eta}^{+\infty-i \eta} \exp \{i \tau(z+t)\} \frac{\mathrm{d} \tau}{\tau^{1+\nu}}=\gamma_{\nu}(z+t)^{\nu} \theta(z+t) \\
& \text { with } \quad \gamma_{\nu}=-\frac{(1+i) i^{\nu+1 / 2} \pi \sqrt{2}}{\Gamma(1+v)}
\end{aligned}
$$

we obtain

$$
R_{v}(z)=3^{-1 / s} \gamma_{\nu} \int_{-z}^{\infty} \operatorname{Ai}\left(3^{-1 / s} t\right)(z+t)^{v} \mathrm{~d} t .
$$

Let us express Airy's function by BESSEL functions ${ }^{26}$ and distinguish the cases $z>0$ and $z \leqq 0$. Setting

we get

$$
\sigma=2(|z| / 3)^{3 / 2} \geqq 0
$$

where

$$
R_{\nu}(z)=3^{v-1} 2^{-(2 v / 3)} \gamma_{\nu} \Lambda_{\nu}^{ \pm}(\sigma)
$$

$$
\begin{aligned}
\Lambda_{\nu}^{+}(\sigma)= & \int_{0}^{\sigma}\left(\sigma^{2 / 3}-u^{2 / 3}\right)^{v}\left(J_{1 / 3}(u)+J_{-1 / 3}(u)\right) \mathrm{d} u \\
& +\frac{\sqrt{3}}{\pi} \int_{0}^{\infty}\left(\sigma^{2 / 3}+u^{2 / 3}\right)^{v} K_{1 / 3}(u) \mathrm{d} u,
\end{aligned}
$$




$$
\Lambda_{v}^{-}(\sigma)=\frac{\sqrt{3}}{\pi} \int_{\sigma}^{\infty}\left(u^{2 / 3}-\sigma^{2 / s}\right)^{v} K_{1 / 3}(u) \mathrm{d} u .
$$

In Eq. (4.13), the positive (negative) sign has to be taken for $z>0(z \leqq 0)$.

Since $K_{1 / 3}(u) \approx(2 /(\pi u))^{1 / 2} \exp \{-u\}$ for $u \gg 1$ the corresponding integrals converge fast. The func. tions $\Lambda_{\nu}^{ \pm}(\sigma)$ have been calculated numerically and are shown in Figs. 1, 2, and 3 for $v=-1 / 2,+1 / 2$,

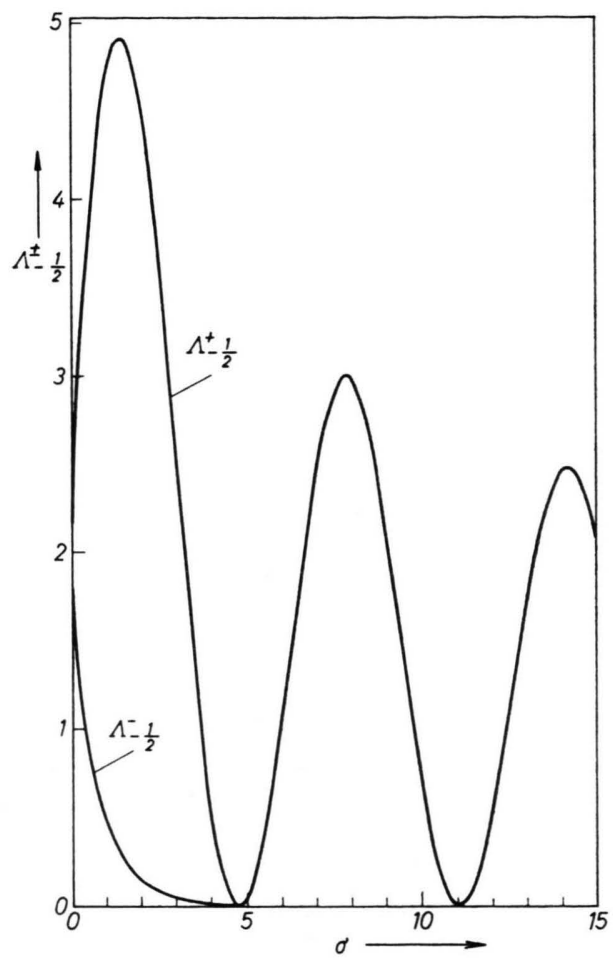

Fig. 1. The Functions $\Lambda_{1 / 2}^{ \pm}(\sigma)$ for $0 \leqq \sigma \leqq 15$, see Eqs. $(4.14 \mathrm{a}, \mathrm{b})$.

$+3 / 2$, respectively. Some values of $\Lambda_{\nu}^{ \pm}(\sigma)$ are given in the Tables 1 and 2 for the range $0 \leqq \sigma \leqq 14$.

Integrating by parts Eq. (4.5) the following recurrence relation can be established

$$
R_{\nu}(z)=(i / v)\left[z R_{\nu-1}(z)-3 R_{\nu-3}(z)\right] .
$$

Using Eq. (4.13) this may be rewritten in terms of $\Lambda_{\nu}^{ \pm}(\sigma)$

$$
\Lambda_{v}^{ \pm}(\sigma)= \pm \sigma^{2 / s} \Lambda_{v-1}^{ \pm}+\frac{4}{9}(v-1)(v-2) \Lambda_{v-3}^{ \pm}
$$

which we shall need in connection with the calculation of the kinetic-energy density.
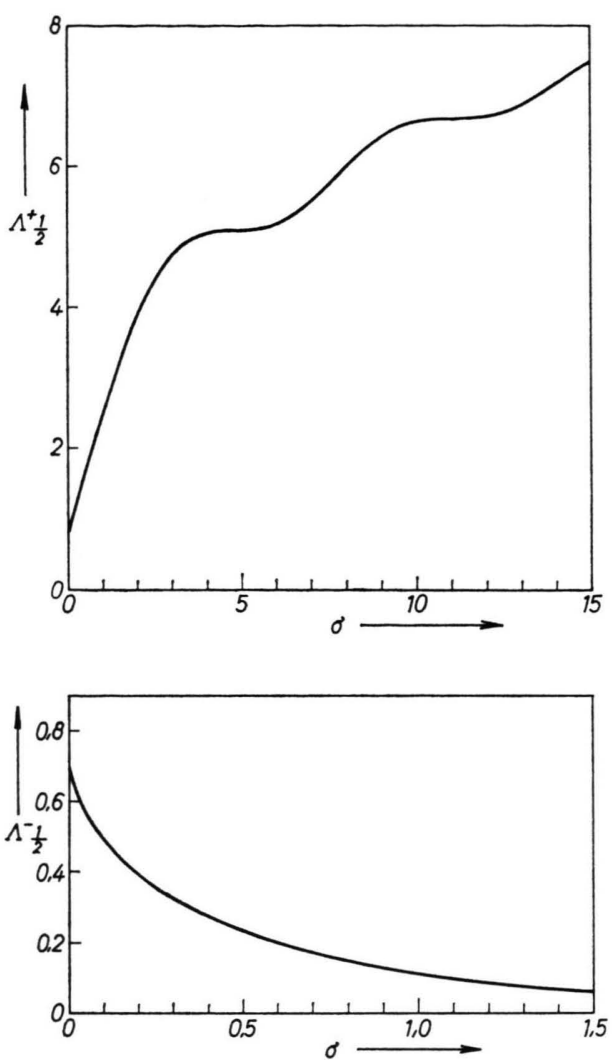

Fig. 2. The Functions $\Lambda_{1 / 2}^{+}(\sigma)$ (upper picture) and $\Lambda_{1 / 2}^{-}(\sigma)$ (lower picture), see Eqs. (4.14 a, b).

Table 1. Values of $\Lambda_{v}{ }^{+}(\sigma)$, Eq. (4.14 a).

\begin{tabular}{rccc}
\hline$\sigma$ & $\Lambda_{-1 / 2}^{+}(\sigma)$ & $\Lambda_{+1 / 2}^{+}(\sigma)$ & $\Lambda_{+3 / 2}^{+}(\sigma)$ \\
\hline 0 & 2.1586 & 0.6969 & 0.5774 \\
1 & 4.7404 & 2.4639 & 2.7777 \\
2 & 4.4624 & 3.8738 & 5.5755 \\
3 & 2.4847 & 4.7539 & 8.7939 \\
4 & 0.5337 & 5.0722 & 12.0587 \\
5 & 0.0479 & 5.1013 & 15.1477 \\
6 & 1.1125 & 5.1923 & 18.0555 \\
7 & 2.5395 & 5.5236 & 20.9162 \\
8 & 2.9786 & 6.0134 & 23.8609 \\
9 & 2.0693 & 6.4412 & 26.9192 \\
10 & 0.6653 & 6.6550 & 30.0204 \\
11 & 0.0003 & 6.6907 & 33.0721 \\
12 & 0.5811 & 6.7196 & 36.0399 \\
13 & 1.7845 & 6.8885 & 38.9657 \\
14 & 2.4708 & 7.1979 & 41.9206 \\
\hline
\end{tabular}

Setting $z=\xi / \zeta$ we obtain from Eqs. (4.3), (4.4), and (4.12)

$$
\sigma=\frac{4}{3}\left(\frac{2 m}{\hbar^{2}}\right)^{1 / 2} \frac{|\lambda-V(\boldsymbol{r})|^{3 / 2}}{|\nabla V(\boldsymbol{r})|}
$$



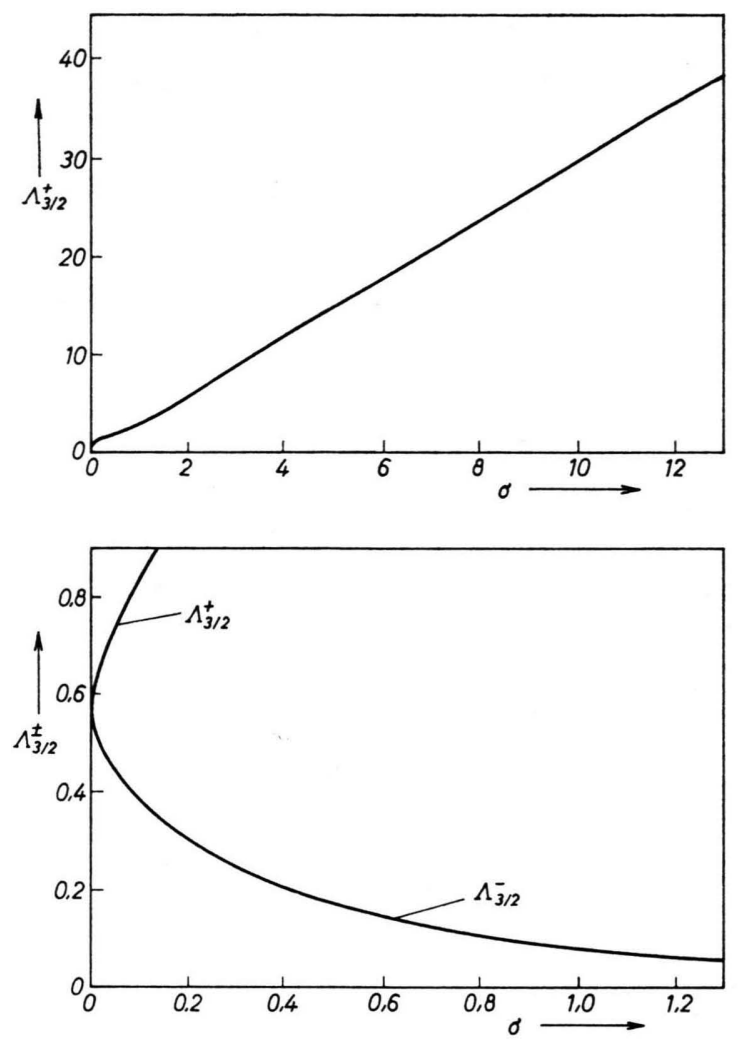

Fig. 3. The Functions $\Lambda_{3 / 2}^{+}(\sigma)$ (upper picture) and $\Lambda_{3 / 2}^{-}(\sigma)$ (lower picture), see Eqs. (4.14 a, b) .

Table 2. Values of $\Lambda_{\nu}{ }^{-}(\sigma)$, Eq. (4.14 b) .

\begin{tabular}{rccc}
\hline$\sigma$ & $\Lambda_{-1 / 2}^{-}(\sigma)$ & $\Lambda_{+1 / 2}^{-}(\sigma)$ & $\Lambda_{+3 / 2}^{-}(\sigma)$ \\
\hline 0 & 2.1586 & 0.6949 & 0.5774 \\
1 & 0.4670 & 0.1137 & 0.07617 \\
2 & 0.1457 & 0.0314 & 0.01908 \\
3 & 0.0481 & 0.00955 & 0.00539 \\
4 & 0.0163 & 0.00303 & 0.00162 \\
5 & 0.00564 & 0.00099 & 0.00050 \\
6 & 0.00196 & 0.00033 & 0.00016 \\
7 & 0.00069 & 0.00011 & $5.2 \cdot 10^{-5}$ \\
8 & $2.4 \cdot 10^{-4}$ & $3.8 \cdot 10^{-5}$ & $1.7 \cdot 10^{-5}$ \\
9 & $8.7 \cdot 10^{-5}$ & $1.3 \cdot 10^{-5}$ & $5.7 \cdot 10^{-6}$ \\
10 & $3.1 \cdot 10^{-5}$ & $4.5 \cdot 10^{-6}$ & $1.9 \cdot 10^{-6}$ \\
11 & $1.1 \cdot 10^{-5}$ & $1.6 \cdot 10^{-6}$ & $6.5 \cdot 10^{-7}$ \\
12 & $3.9 \cdot 10^{-6}$ & $5 \cdot 10^{-7}$ & $2.2 \cdot 10^{-7}$ \\
13 & $1.4 \cdot 10^{-6}$ & $2 \cdot 10^{-7}$ & $7 \cdot 10^{-8}$ \\
14 & $5 \cdot 10^{-7}$ & $7 \cdot 10^{-8}$ & $2 \cdot 10^{-8}$ \\
\hline
\end{tabular}

and from Eqs. (3.11), (4.3), (4.4), (4.6), (4.7), (4.10), and (4.13) it follows

$$
\varrho(\boldsymbol{r})=\frac{m|\nabla V(\boldsymbol{r})|}{6 \hbar^{2} \pi^{2}} \Lambda_{3 / 2}^{ \pm}(\sigma),
$$

$\nabla \varrho(\boldsymbol{r})=-\frac{(2 m)^{4 / 3}}{2 \pi^{2} 6^{2 / 3} \hbar^{8 / 3}} \nabla V(\boldsymbol{r})|\nabla V(\boldsymbol{r})|^{1 / 3} \Lambda_{1 / 2}^{ \pm}(\sigma)$.
Here we have to take the positive (negative) sign for $\lambda-V>0(\leqq 0)$. Since $\Lambda_{3 / 2}^{ \pm}(\sigma) \geqq 0$ for all $\sigma$ (see Fig. 3) the density cannot become negative.

\section{Density of the Kinetic Energy as a Function of $V(\boldsymbol{r})$ and $\nabla V(\boldsymbol{r})$}

The ground state kinetic energy of our system of independent electrons is given in coordinate representation by

$$
\begin{aligned}
& 2 \sum_{r=1}^{n} \sum_{\mu=1}^{m(r)}\left\langle\psi_{r, u}\left|\frac{\hat{\boldsymbol{p}}^{2}}{2 m}\right| \psi_{r, \mu}\right\rangle \\
&=\int\left\{\frac{\hbar^{2}}{m} \sum_{r=1}^{n} \sum_{\mu=1}^{m(r)}\left|\nabla \psi_{r, \mu}\right|^{2}\right\} \mathrm{d}^{3} \boldsymbol{r}
\end{aligned}
$$

where the factor 2 is due to the two spin orientations belonging to each $\psi_{r, \mu}$. The integrand on the right is regarded as the density $\varepsilon_{\mathrm{k}}$ of the total kinetic energy. Evidently, $\varepsilon_{\mathrm{k}}$ is positive definite in contrast to the usual definition

$$
\varepsilon_{\mathrm{k}}^{\prime} \equiv-\frac{\hbar^{2}}{2 m} 2 \sum_{r=1}^{n} \sum_{\mu=1}^{m(r)} \psi_{r, \mu}^{*} \Delta \psi_{r, \mu} .
$$

The difference between $\varepsilon_{\mathrm{k}}$ and $\varepsilon_{\mathrm{k}}{ }^{\prime}$ is a divergence term. Since the kinetic energy itself is always positive it is convenient to choose the corresponding density positive definite too.

In terms of Dirac's density matrix, Eq. (2.3), $\varepsilon_{\mathrm{k}}$ may be written

$$
\varepsilon_{\mathrm{k}}=\frac{\hbar^{2}}{2 m} \sum_{i=1}^{3}\left[\frac{\partial^{2}}{\partial x_{i} \partial x_{i}{ }^{\prime}} \varrho\left(\boldsymbol{r}, \boldsymbol{r}^{\prime}\right)\right]_{\boldsymbol{r}^{\prime}=\boldsymbol{r}} .
$$

This expression is now evaluated on the basis of the linear potential approximation, Eq. (3.1). Thus we start from Eqs. (3.10), (3.11), (3.12 a, b, c) for the density matrix.

For infinitesimal $\left|\boldsymbol{r}-\boldsymbol{r}^{\prime}\right|$, it is correct within our approximation to set $\nabla V\left(\boldsymbol{r}^{\prime}\right)=\nabla V(\boldsymbol{r})$. Thus

$$
\varrho\left(\boldsymbol{r}, \boldsymbol{r}^{\prime}\right)=C \int_{-\infty i \eta}^{+\infty} \frac{\mathrm{d} \omega}{\omega / \mathrm{s} / \mathrm{z}} \exp \left\{i K\left(\boldsymbol{r}, \boldsymbol{r}^{\prime}, \omega\right)\right\}
$$

where

$$
\begin{array}{r}
K\left(\boldsymbol{r}, \boldsymbol{r}^{\prime}, \omega\right)=\omega\left[\lambda-\frac{1}{2}\left\{V(\boldsymbol{r})+V\left(\boldsymbol{r}^{\prime}\right)\right\}\right]+\frac{m\left(\boldsymbol{r}-\boldsymbol{r}^{\prime}\right)^{2}}{2 \hbar^{2} \omega} \\
-\frac{\hbar^{2} \omega^{3}}{24 m} \cdot|\nabla V(\boldsymbol{r})|^{2} \cdot(5.5)
\end{array}
$$

Differentiating $\varrho\left(\boldsymbol{r}, \boldsymbol{r}^{\prime}\right)$ with respect to $x_{i}$ and $x_{i}^{\prime}$ under the integral sign and then setting $\boldsymbol{r}^{\prime}=\boldsymbol{r}$ we 
find from Eq. (5.3)

$$
\begin{gathered}
\frac{2 m}{\hbar^{2}} \varepsilon_{\mathrm{k}}=-\frac{3 i m \stackrel{+}{C}}{\hbar^{2}} \int_{-\infty i \eta}^{\infty-i \eta} \frac{\mathrm{d} \omega}{\omega^{7 / 2}} \exp \{i K(\boldsymbol{r}, \boldsymbol{r}, \omega)\} \\
-\frac{1}{4} C|\nabla V(\boldsymbol{r})|_{-\infty}^{+\infty} \int_{-i \eta}^{\infty-i \eta} \frac{\mathrm{d} \omega}{\omega^{1 / 2}} \exp \{i K(\boldsymbol{r}, \boldsymbol{r}, \omega)\} .
\end{gathered}
$$

Using Eqs. (4.3), (4.4), (4.5), (4.12), (4.13), and $(4.14, \mathrm{a}, \mathrm{b})$ we get

$$
\begin{gathered}
K(\boldsymbol{r}, \boldsymbol{r}, \omega)=\omega \xi-\omega^{3} \zeta^{3} \\
\int_{-\infty-i \eta}^{+\infty-i \eta} \frac{\mathrm{d} \omega}{\omega^{1+\nu}} \exp \{i K(\boldsymbol{r}, \boldsymbol{r}, \omega)\}=\zeta^{\nu} R_{\nu}(\xi / \zeta) \\
=3^{\nu-1} 2^{-(2 \nu / 3)} \gamma_{\nu} \zeta^{\nu} \Lambda_{\nu}^{ \pm}(\sigma) .
\end{gathered}
$$

In terms of $\Lambda_{v}^{ \pm}(\sigma)$ we thus find

$$
\varepsilon_{\mathrm{k}}=\left(\frac{2 m}{\hbar^{2}}\right)^{2 / 3} \frac{|\nabla V|^{5 / 3}}{8 \pi^{2} 6^{1 / 3}}\left(\frac{3}{5} \Lambda_{5 / 2}^{ \pm}(\sigma)+\frac{1}{3} \Lambda_{-1 / 2}^{ \pm}(\sigma)\right) .
$$

Applying the recurrence relation (4.15a) we eliminate $A_{5 / 2}^{ \pm}$

$\varepsilon_{\mathrm{k}}=\left(\frac{2 m}{\hbar^{2}}\right)^{2 / 3} \frac{|\nabla V|^{5 / 3}}{8 \pi^{2} 6^{1 / 3}}\left\{ \pm \frac{3}{5} \sigma^{2 / 3} \Lambda_{3 / 2}^{ \pm}(\sigma)+\frac{8}{15} \Lambda_{-1 / 2}^{ \pm}(\sigma)\right\}$.

Since the positive (negative) sign has to be taken for $\lambda-V>0(\leqq 0)$ it follows from Eq. (4.16)

$$
\begin{aligned}
\pm \sigma^{2 / 3} & = \pm|\lambda-V(\boldsymbol{r})|\left(\frac{4(2 m)^{1 / 2}}{3 \hbar|\nabla V|}\right)^{2 / 3} \\
& =(\lambda-V(\boldsymbol{r}))\left(\frac{4(2 m)^{1 / 2}}{3 \hbar|\nabla V|}\right)^{2 / 3} .
\end{aligned}
$$

Thus $\varepsilon_{\mathrm{k}}$ can be written

$$
\begin{aligned}
\varepsilon_{\mathrm{k}}=\frac{m|\nabla V|}{10 \pi^{2} \hbar^{2}} \cdot(\lambda-V(\boldsymbol{r})) \cdot \Lambda_{3 / 2}^{ \pm}(\sigma) \\
\quad+\left(\frac{2 m}{\hbar^{2}}\right)^{2 / 3} \frac{\mid \nabla V V^{5 / 3}}{15 \pi^{2} 6^{1 / 3}} \cdot \Lambda_{-1 / 2}^{ \pm}(\sigma) .
\end{aligned}
$$

\section{The Kinetic-Energy Density as a Function of $\varrho$ and $\nabla \varrho$}

Having derived expressions for $\varrho, \nabla \varrho$, and $\varepsilon_{\mathrm{k}}$ as functions of the potential and of its gradient, we shall now eliminate $\lambda-V$ and $\nabla V$ in order to obtain $\varepsilon_{\mathrm{k}}$ as a function of $\varrho$ and of $\nabla \varrho$.

From Eqs. (4.16), (4.17), and (4.18) we obtain

$$
\begin{gathered}
y \equiv \frac{|\nabla \varrho|^{1 / 2}}{\varrho^{2 / s}}=2^{1 / 2}(3 \pi)^{1 / 3} \frac{\left[\Lambda_{1 / 2}^{ \pm}(\sigma)\right]^{1 / 2}}{\left[\Lambda_{3 / 2}^{ \pm}(\sigma)\right]^{2 / 3}}, \\
|\nabla V|=\frac{6 \hbar^{2} \pi^{2} \varrho}{m \Lambda_{3 / 2}^{ \pm}(\sigma)},
\end{gathered}
$$

$$
\lambda-V(\boldsymbol{r})= \pm|\lambda-V(\boldsymbol{r})|= \pm \frac{\hbar^{2}(3 \pi)^{4 / 3}}{2 m}\left(\frac{\sigma \varrho}{\Lambda_{3 / 2}^{ \pm}(\sigma)}\right)^{2 / 3}
$$

Substitution of Eqs. (6.2) and (6.3) into Eq. (5.12) yields $\varepsilon_{\mathrm{k}}$ as a product of the kinetic-energy density in $\mathrm{TF}$-approximation and a variable correction factor

where

$$
\varepsilon_{\mathrm{k}}=\varepsilon_{\mathrm{k}}^{\mathrm{TF}} \cdot \chi(\sigma)
$$

$\varepsilon_{\mathrm{k}}^{\mathrm{TF}}=\frac{3}{5}\left(3 \pi^{2}\right)^{2 / 3} \frac{\hbar^{2}}{2 m} \varrho^{5 / 3}=\varkappa \varrho^{5 / 3}$

and

$$
\chi(\sigma)=\left(\frac{3}{\Lambda_{3 / 2}^{ \pm}(\sigma)}\right)^{2 / 3}\left(\frac{8}{9} \frac{\Lambda_{-1 / 2}^{ \pm}(\sigma)}{\Lambda_{3 / 2}^{ \pm}(\sigma)} \pm \sigma^{2 / 3}\right) .
$$

The last step to be done is to solve Eq. (6.1) for $\sigma$ and insert the solution $\sigma=\sigma(y)=\sigma\left(|\nabla \varrho|^{1 / 2} \varrho^{-2 / 3}\right)$ into Eq. (6.6). We thus arrive at the wanted relation between $\varepsilon_{\mathrm{k}}, \varrho$, and $\nabla \varrho$ :

$$
\varepsilon_{\mathrm{k}}=\varkappa \varrho^{5 / 3} \chi(\sigma(y))=\varkappa \varrho^{5 / 3} \bar{\chi}\left(|\nabla \varrho|^{1 / 2} \varrho^{-2 / s}\right) .
$$

The function $y(\sigma)$, Eq. (6.1), has been computed numerically; it is shown in Figure 4 . From the asymptotic behaviour of $y(\sigma)$, see Eqs. (7.1) and (7.7), and from Fig. 4 it is clear that $\sigma$ and

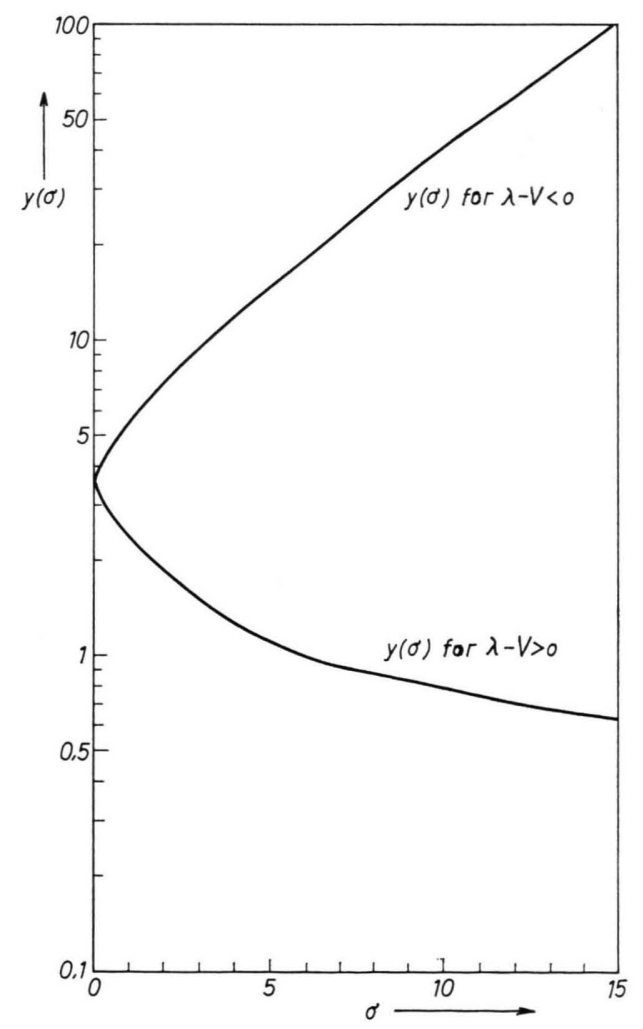

Fig. 4. The Function $y(\sigma)$ for both positive and negative values of $\lambda-V$, see Eq. (6.1). 
$\operatorname{sign}(\lambda-V)$ are determined uniquely by $y$. Then, according to Eq. (6.6), also $\chi$ is determined unambiguously by $y$, i. e., $\bar{\chi}$ defined by Eq. (6.7) is a unique function of $y$. However, it doesn't seem possible to eliminate $\sigma$ and $\operatorname{sign}(\lambda-V)$ in an analytical way except for the limits $\sigma \rightarrow \infty ; \operatorname{sign}(\lambda-V)$ $= \pm 1$ which shall be treated in the next section. For general values of $\sigma$ we must be satisfied with numerical evaluation of $\bar{\chi}(y)$, which is given as a parametric representation $y=y(\sigma, j) ; \chi=\chi(\sigma, j)$ with the continuous parameter $\sigma$ and the discrete parameter $j=\operatorname{sign}(\lambda-V)$. Some values of $\bar{\chi}(y)$ are listed in Table 3 . Figures $5 \mathrm{a}, \mathrm{b}$ show this function for different ranges of $y$.

Table 3. Values of $\bar{\chi}(y)$. Eq. (6.7).

\begin{tabular}{llcc}
\hline$y$ & $\bar{\chi}(y)$ & $y$ & $\bar{\chi}(y)$ \\
\hline 0 & 1 & 2.8 & 4.32256 \\
0.6 & 0.999407 & 3.0 & 5.39648 \\
0.8 & 1.00427 & 3.5 & 9.10154 \\
1.0 & 1.01154 & 4.0 & 14.6539 \\
1.2 & 0.996981 & 4.5 & 22.5701 \\
1.4 & 1.05822 & 5.0 & 33.4471 \\
1.6 & 1.19679 & 6.0 & 66.8190 \\
1.8 & 1.42045 & 7.0 & 120.911 \\
2.0 & 1.74092 & 8.0 & 202.951 \\
2.2 & 2.17289 & 9.0 & 321.243 \\
2.4 & 2.73364 & 10.0 & 485.185 \\
2.6 & 3.44295 & & \\
\hline
\end{tabular}

First of all, we note that $\bar{\chi}(y)$ is positive for all $y(\geqq 0)$. Therefore, according to Eq. (6.4), $\varepsilon_{\mathrm{k}}$ never becomes negative and vanishes only for $\varrho=0$, i. e. $\varepsilon_{\mathrm{k}}$ is positive definite.

For $y=0$ which means either $\varrho \rightarrow \infty$ or/and $\nabla \varrho=0$ we have

$$
\begin{array}{cc} 
& \bar{\chi}(0)=1 \\
\text { or } & \varepsilon_{\mathrm{k}}=\varkappa \varrho^{5 / 3}=\varepsilon_{\mathrm{k}}^{\mathrm{TF}}
\end{array}
$$

as is expected. However, we also obtain oscillations of decreasing wavelength and amplitude when $y$ approaches zero.

If $\varrho \ll|\nabla \varrho|^{3 / 4}$ or $y \gg 1, \bar{\chi}(y)$ becomes large compared with unity, thus

$$
\varkappa \varrho^{5 / 3} / \varepsilon_{\mathrm{k}}=1 / \bar{\chi}(y) \ll 1 .
$$

The TF-fraction to the whole kinetic-energy density is small for large $y$.

The quantitative behaviour of $\bar{\chi}(y)$ for $y \rightarrow 0$ and $y \rightarrow \infty$ is investigated in Section 7 .

For comparison, Figs. 5 a. b also show $\bar{\chi}^{\mathrm{TF}}(y) \equiv \mathbf{1}$ and $\bar{\chi}^{\mathrm{TFW}}(y)$. The latter corresponds to the Thomas-

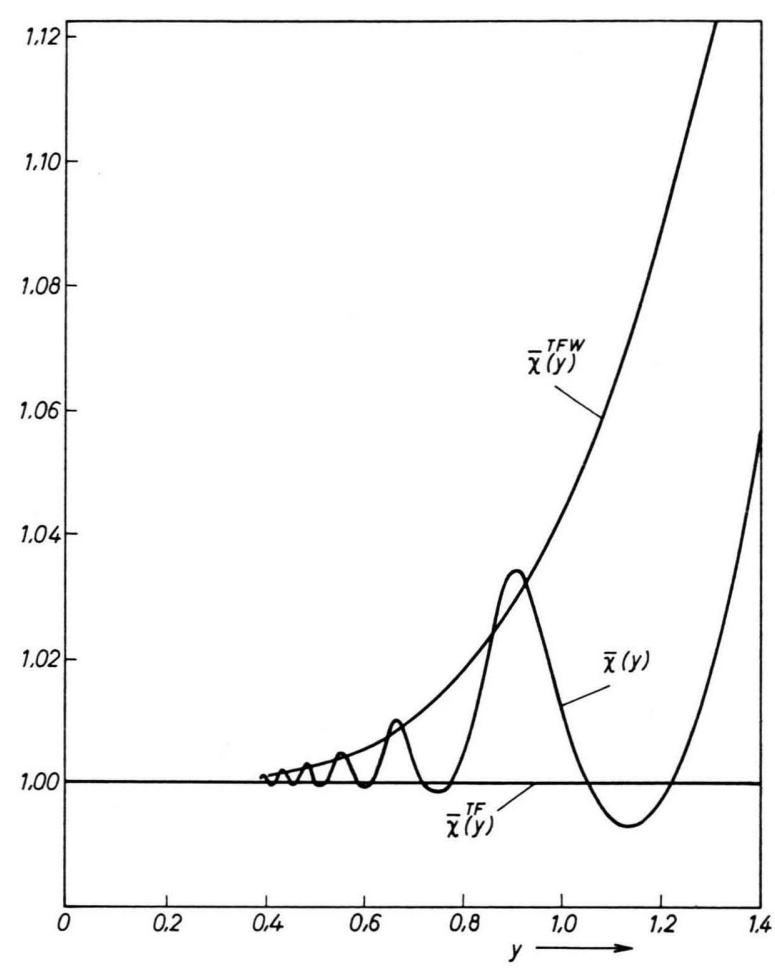

Fig. 5 a. The Functions $\bar{\chi}(y), \bar{\chi}^{\mathrm{TF}}(y)$, and $\bar{\chi}^{\mathrm{TFW}}(y)$ in the range $0.4 \leqq y \leqq 1.4$, see Eqs. (6.7), (6.11).

Fermi-Weizsäcker energy-density functional which is given by

$$
\varepsilon_{\mathrm{k}}^{\mathrm{TFW}}=\varkappa \varrho^{5 / 3}+\frac{\hbar^{2}}{8 m} \frac{(\nabla \varrho)^{2}}{\varrho} .
$$

Using the definition of $y$, Eq. $(6.1), \varepsilon_{\mathrm{k}}^{\mathrm{TFW}}$ can be rewritten as

$$
\varepsilon_{\mathrm{k}}^{\mathrm{TFW}}=\varkappa \varrho^{5 / 3}\left(1+\frac{5}{1 \subseteq}\left(3 \pi^{2}\right)^{-2 / 3} y^{4}\right)=\varepsilon_{\mathrm{k}}^{\mathrm{TF}} \cdot \bar{\chi}^{\mathrm{TFW}}(y) .
$$

\section{Asymptotic Behaviour of $\varepsilon_{\mathrm{k}}$ in the Limits}

$$
y \rightarrow 0 \text { and } y \rightarrow \infty
$$

$$
\text { 7.1. The Limit } y \rightarrow 0
$$

If $\varrho$ is large and/or $\nabla \varrho \rightarrow 0 \quad y$ becomes small which implies $\sigma \rightarrow \infty$ and $\lambda-V>0$. From Eqs. (6.1) and $(A 7 a, b)$ we obtain

$$
y=a \sigma^{-1 / 2}\left\{1-\frac{\cos \sigma}{6 \sigma}+O\left(\sigma^{-2 / 3}\right)\right\}
$$

with

$$
a=2^{1 / 2}\left(3 \pi^{2}\right)^{1 / 6} .
$$

Let us solve Eq. (7.1) for $\sigma$ by iteration. In zeroth order we find $y \approx a \sigma^{-1 / 2}$ or $\sigma \approx(a / y)^{2}$. Inserting 


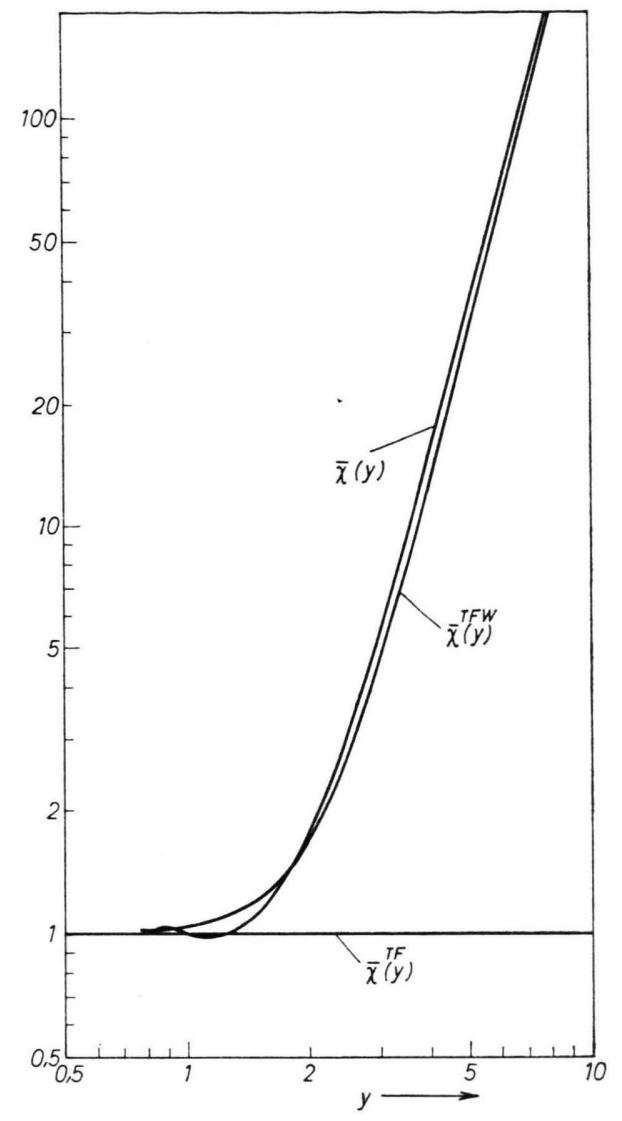

Fig. 5 b. The Functions $\bar{\chi}(y), \bar{\chi}^{\mathrm{TF}}(y)$, and $\bar{\chi}^{\mathrm{TFW}}(y)$ in the range $0.8 \leqq y \leqq 8$, see Eqs. (6.7), (6.11).

this into the oscillatinig term of Eq. (7.1) we get in next approximation

$$
\sigma \approx\left(\frac{a}{y}\right)^{2}\left\{1-\frac{1}{3}\left(\frac{y}{a}\right)^{2} \cdot \cos \left(\frac{a}{y}\right)^{2}\right\} .
$$

The asymptotic expansion of $\chi(\sigma)$ for $\lambda-V>0$ follows from Eqs. (6.6) and (A7a,c)

$$
\chi(\sigma)=1+\frac{5}{9 \sigma^{2}}\left(\frac{5}{3}+2 \sin \sigma\right)+O\left(\sigma^{-5 / 2}\right) .
$$

Substitution of expression (7.3) yields

$\bar{\chi}(y)=1+\frac{5}{9}\left(\begin{array}{c}y \\ a\end{array}\right)^{4}\left\{\frac{5}{3}+2 \sin \sigma(y)\right\}+O\left(y^{5}\right)$

Thus, for small $|\nabla \varrho|^{1 / 2} \varrho^{-2 / 3}, \varepsilon_{\mathrm{k}}$ is given by

$$
\begin{aligned}
\varepsilon_{\mathrm{k}}=\varepsilon_{\mathrm{k}}^{\mathrm{TF}} & +\frac{5}{9} \hbar^{2} \frac{(\nabla \varrho)^{2}}{\varrho} \\
& +\frac{2}{3} \frac{\hbar^{2}}{8 \mathrm{~m}} \underset{(\nabla \varrho)^{2}}{\varrho} \sin \left\{\begin{array}{c}
2\left(3 \pi^{2}\right)^{1 / 3} \varrho^{4 / 3} \\
|\nabla \varrho|
\end{array}\right\} .
\end{aligned}
$$

\subsection{The Limit $y \rightarrow \infty$}

The case $y \rightarrow \infty$ which corresponds to $|\nabla \varrho| \gg \varrho^{4 / 3}$ implies $\sigma \rightarrow \infty$ and $\lambda-V<0$. Using Eqs. (Alla, b,c) we obtain from (6.1)

$$
y=(12 \pi)^{1 / 3} \sigma^{1 / 3} e^{\sigma / 6}
$$

and from Eq. (6.6)

$$
\chi(\sigma)=\frac{5}{3} 6^{2 / 3}\left(\sigma^{1 / 3} e^{\sigma / 6}\right)^{4} .
$$

Hence we get

$$
\chi(\sigma(y))=\bar{\chi}(y)=\frac{5}{1}\left(3 \pi^{2}\right)^{-2 / 3} y^{4}
$$

which shows that $\bar{\chi}(y)$ equals $\bar{\chi}^{\mathrm{TWF}}(y)$ asymptotically for large $y$, see Equation (6.11). Finally, the kinetic-energy density becomes

$$
\varepsilon_{\mathrm{k}}=\frac{\hbar^{2}}{8 m} \frac{|\nabla \varrho|^{2}}{\varrho} .
$$

\section{Conclusion}

The kinetic-energy density functional $\varepsilon_{\mathrm{k}}$ based upon the approximation of locally linear potential ad given by Eqs. (6.1), (6.4), (6.5), and (6.6) covers the whole range between very high and very low density.

For small $\varrho$ or high $\nabla \varrho$ such that

$$
y=|\nabla \varrho|^{1 / 2} \varrho^{-2 / s} \gg 1
$$

the main contribution to $\varepsilon_{\mathrm{k}}$ is given by the Weizsäcker correction term alone, see Equation (7.10).

If $y \ll 1$ corresponding to a weakly inhomogeneous electron gas, $\varepsilon_{\mathrm{k}}$ is approximately given by Equation (7.6). This result differs from the Kirzhnits approximation in two points:

(a) The factor of $5 / 9$ at the steady gradient correction term lies just in the middle between 1 (full Weizsäcker term) and $1 / 9$ (Kirzhnits term). When KIRZHNITS' calculation ${ }^{27}$ is repeated using, however, the positive definite expression (5.3) for $\varepsilon_{k}$ and neglecting all derivatives of $V$ higher than the first, then our factor of $5 / 9$ is recovered.

(b) The third term in Eq. (7.6) representing quantum oscillations which are missing in Kirzhnits' work is of same order of magnitude as the second one. When $|\nabla \varrho| \rightarrow 0$, also the oscillating term approaches zero. However, the number of oscillations becomes infinite which is unphysical, of course, provided that this limit is reached within a finite domain of space. Now, if $V$ is exactly linear with $\nabla V \neq 0$ it follows from $\varrho^{4 / 3}|\nabla \varrho|^{-1} \sim y^{-2} \sim \sigma$ [see 
Eq. (7.3)] and from the definition of $\sigma$, Eq. (4.16), that the limit $\nabla \varrho \rightarrow 0$ implies $\lambda-V \rightarrow \infty$ which is clearly impossible within a finite region. Since, however, each potential which is capable of bound states cannot be exactly linear over the whole space those unphysical accumulation of oscillations may appear within finite regions.

From these considerations the author hopes that if the approximation scheme presented here is extended to include the second derivatives of $V$, one should be able to reproduce

(1) the steady correction term of Kirzhnits in the limit of weak slowly varying inhomogeneity,

(2) the Weizsäcker result in the limit of small $\varrho$ or large $\nabla \varrho$,

(3) oscillatory behaviour of $\varepsilon_{\mathrm{k}}$ which is reasonable also quantitatively.

Investigations in this direction are in progress.

\section{Acknowledgments}

The author wishes to thank Professor Dr. G. Simon for suggesting this work and for many helpful discussions. Thanks are also due to Professor Dr. A. M. K. MüLLER for numerous valuable discussions.

\section{Appendix}

\section{The Asymptotic Behaviour of the Functions $\Lambda_{v}^{ \pm}(\sigma)$}

The rather lengthy calculation of the asymptotic form of $\Lambda_{v}^{ \pm}(\sigma)$, Eqs. (4.14 a, b), can only be sketched here.

First, let us consider $\Lambda_{v}^{+}(\sigma)$ which may be written as the sum of two integrals

$W_{\nu}(\sigma)=\sigma^{2 v / 3} \int_{0}^{\sigma}\left(1-\left(\frac{u}{\sigma}\right)^{2 / 3}\right)^{v}\left(J_{1 / 3}(u)+J_{-1 / 3}(u)\right) \mathrm{d} u$,

$L_{\nu}(\sigma)=\frac{\sqrt{3}}{\pi} \sigma^{2 \nu / 3} \int_{0}^{\infty}\left(1+\left(\frac{u}{\sigma}\right)^{2 / 3}\right)^{\nu} K_{1 / 3}(u) \mathrm{d} u$.

Since in $W_{\nu}(\sigma)$ an expansion of $\left(1-(u / \sigma)^{2 / s}\right)^{\nu}$ in powers of $(u / \sigma)^{2 / s}$ diverges at the upper limit $u=\sigma$ we divide the interval of integration into $I_{1} \equiv[0, \theta \sigma]$ and $I_{2} \equiv[\theta \sigma, \sigma]$ with $0<\theta<1$ and use the expansion in $I_{1}$ only. If $\sigma$ is large enough we have also $\theta \sigma \gg 1$. Therefore, we may integrate termwise over $I_{1}$ using a relation for integrals of Bessel functions with large upper limits ${ }^{29}$. In the integral over $I_{2}$ we substitute $u(v)=\sigma\left(1-v^{2}\right)^{2 / 3}$ and replace the Bessel functions by their asymptotic expansions. The asymptotic forms of the arising integrals may be found by repeated integration by parts and by applying the method of stationary phase ${ }^{30}$. The result is

$$
\begin{gathered}
W_{3 / 2}(\sigma)=2 \sigma-\frac{3 \sigma^{1 / 3}}{2^{1 / 3} \Gamma\left(\frac{1}{3}\right)}-4 \Gamma\left(\frac{2}{3}\right) \sigma^{1 / 3} \\
-\frac{1}{9 \sigma}-\frac{\sin \sigma}{\sigma}+O\left(\sigma^{-3 / 2}\right) \\
W_{1 / 2}(\sigma)=2 \sigma^{1 / 3}-\frac{1}{2^{1 / 3} \Gamma\left(\frac{1}{3}\right) \sigma^{1 / 3}}+\frac{2^{1 / 3}}{12 \Gamma\left(\frac{2}{3}\right) \sigma} \\
-\frac{\cos \sigma}{\sigma^{2 / 3}}+O\left(\sigma^{-7 / 6}\right) \\
W_{-1 / 2}(\sigma)=\frac{2+3 \sin \sigma}{\sigma^{1 / 3}}+O\left(\sigma^{-5 / 6}\right) .
\end{gathered}
$$

Denoting the integrand of $L_{v}(\sigma)$, Eq. (A2), with $f(u, \sigma, v)$ let us split the region of integration

$$
\begin{aligned}
L_{v}(\sigma) & =L_{1, v}(\sigma, \theta)+L_{2, v}(\sigma, \theta), \\
L_{1, v}(\sigma, \theta) & =\int_{0}^{\vartheta \sigma} f(u, \sigma, v) \mathrm{d} u, \\
L_{2, v}(\sigma, \theta) & =\int_{\vartheta \sigma}^{\infty} f(u, \sigma, v) \mathrm{d} u
\end{aligned}
$$

with $0<\theta<1$. In $L_{2, v}$ we may replace $K_{1 / s}$ by its asymptotic form if $\theta \sigma$ is large enough. Then it is estimated easily that $L_{2, \nu}$ falls off roughly as $\exp \{-\theta \sigma\}$ for all $\nu$. In $L_{1, \nu}$ we may expand

$$
\left(1+\left(\frac{u}{\sigma}\right)^{2 / 3}\right)^{\nu}=\sum_{\mu=0}^{\infty}\left(\begin{array}{l}
v \\
\mu
\end{array}\right)\left(\frac{u}{\sigma}\right)^{2 \mu / 3}
$$

and split up $\int_{0}^{\vartheta \sigma}=\int_{0}^{\infty}-\int_{\vartheta \sigma}^{\infty}$. The first integral is integrated exactly, term by term, while the second can be shown to fall off $\sim \exp \{-\theta \sigma\}$ due to the asymptotic behaviour of $K_{1 / 3}$. Neglecting all terms with exponential decay we find

$$
\begin{gathered}
L_{3 / 2}(\sigma)=\sigma+\frac{3 \sigma^{1 / 3}}{2^{1 / 3} \Gamma\left(\frac{1}{3}\right)}+\frac{2^{1 / 3}}{4 \Gamma\left(\frac{2}{3}\right) \sigma^{1 / 3}} \\
-\frac{1}{18 \sigma}+O\left(\sigma^{-5 / 3}\right), \quad(\mathrm{A} 6 \mathrm{a}) \\
L_{1 / 2}(\sigma)=\sigma^{1 / 3}+\frac{1}{2^{1 / 3} \Gamma\left(\frac{1}{3}\right) \sigma^{1 / 3}}-\frac{2^{1 / 3}}{12 \Gamma\left(\frac{2}{3}\right) \sigma}+O\left(\sigma^{-5 / 3}\right), \\
\quad(\mathrm{A} 6 \mathrm{~b}) \\
L_{-1 / 2}(\sigma)=\sigma^{-1 / 3}-\frac{1}{2^{1 / 3} \Gamma\left(\frac{1}{3}\right) \sigma}+O\left(\sigma^{-5 / 3}\right) .
\end{gathered}
$$

Combining Eqs. (4.14a), (A1), (A2), and (A3a-c) we thus arrive at

$\Lambda_{3 / 2}^{+}(\sigma)=3 \sigma-1 /(6 \sigma)-\sin \sigma / \sigma+O\left(\sigma^{-3 / 2}\right)$, (A7a) 
$\Lambda_{1 / 2}^{+}(\sigma)=3 \sigma^{1 / 3}-\cos \sigma / \sigma^{2 / 3}+O\left(\sigma^{-7 / 8}\right)$,

$\Lambda_{-1 / 2}^{+}(\sigma)=3 \sigma^{-1 / 3}(1+\sin \sigma)+O\left(\sigma^{-5 / 6}\right)$.

Concerning $\Lambda_{v}^{-}(\sigma)$ we need only the leading terms of the asymptotic expansions. Setting as a new variable of integration $v=u / \sigma-1$ we obtain from Equation $(4.14 \mathrm{~b})$

$$
\Lambda_{v}^{-}(\sigma)=g_{v}(\sigma) \int_{0}^{\infty} e^{-\sigma v} h_{v}(v) \mathrm{d} v
$$

where

$$
g_{\nu}(\sigma)=\left(\frac{3}{2 \pi}\right)^{1 / 2} \sigma^{(2 v / 3+1 / 2)} e^{-\sigma}
$$

1 P. Hohenberg and W. Kohn, Phys. Rev. 136, B 864 [1964].

2 C. F. v. WeIzsäcker, Z. Phys. 96, 431 [1935].

3 R. Berg and L. Wilets, Proc. Phys. Soc., London A 68, 229 [1955].

4 K. Yonei and Y. Tomishima, J. Phys. Soc. Japan 20, 1051 [1965].

5 Y. Tomishima and K. Yonei, J. Phys. Soc. Japan 21, 142 [1966].

6 D. A. Kirzhnits, Zh. Eksperim. Teor. Fiz. 32, 115 [1957], in Russian. English in: Sov. Phys. JETP 5, 64 [1957].

7 A. S. Kompaneets and E. S. PavlovskiI, J. Exp. Theor. Phys. 4, 328 [1957].

8 K. L. Le Couteur, Proc. Phys. Soc. London 84, 837 [1964].

9 J. C. Stoddart, A. M. Beattie, and N. H. March, Int. J. Quantum Chem. 4 S, 35 [1970].

10 S. Golden, Phys. Rev. 105, 604 [1957].

11 P. Gombás, Acta Phys. Hung. 5, 483 [1956].

12 P. Gombás, Ann. Phys. (6) 18, 1 [1956].

13 P. Gombás, Acta Phys. Hung. 25, 361 [1968].

14 M. J. Stephen and K. Zalewski, Proc. Roy. Soc. London A 270, 435 [1962].

15 H. PAyne, Phys. Rev. 132, 2544 [1963].

$$
h_{v}(v)=(1+v)^{-1 / 2}\left\{(1+v)^{2 / 3}-1\right\}^{v} \cdot(\mathrm{A} 10)
$$

Since $\int_{0}^{\infty} v^{\gamma} e^{-\sigma v} \mathrm{~d} v=\sigma^{-\gamma-1} \Gamma(1+\gamma)$ the leading term of $A_{v}^{-}(\sigma)$ is determined by the lowest order term $(2 v / 3)^{v}$ in the power series expansion of $h_{v}(v)$. Thus we get

$$
\text { or } \begin{aligned}
\Lambda_{v}^{-}(\sigma) & =g_{v}(\sigma) \\
& \int_{0}^{\infty} e^{-\sigma v}(2 v / 3)^{v} \mathrm{~d} v \\
& \Lambda_{3 / 2}^{-}(\sigma)=\frac{1}{2} \sigma^{-1} e^{-\sigma} \\
& \Lambda_{1 / 2}^{-}(\sigma)=\frac{1}{2} \sigma^{-2 / s} e^{-\sigma} \\
& \Lambda_{-1 / 2}^{-}(\sigma)=\frac{3}{2} \sigma^{-1 / s} e^{-\sigma} .
\end{aligned}
$$

16 N. L. Balazs, Phys. Rev. 134, A 841 [1964].

17 P. Gombás, Phys. Lett. 28 A, 585 [1969].

18 H. M. Schey and Y. L. SchwartZ, Phys. Rev. 137, A 709 [1965].

19 W. Jones and W. H. Young, J. Phys. C 4, 1322 [1971].

20 J. C. Stoddart and N. H. March, Proc. Roy. Soc. London A 299, 279 [1967].

21 W. J. SwiATECKI, Proc. Phys. Soc. London A 68, 285 [1955].

22 L. C. Alfred, Phys. Rev. 121, 1275 [1961].

23 W. Macke and P. Rennert, Ann. Phys. 12, 84 [1963].

24 R. M. Wilcox, J. Math. Phys. 8, 962 [1967].

25 M. Abramowitz and I. A. Stegun, Handbook of Mathematical Functions, Dover, New York 1965.

26 loc. cit., p. 447.

27 D. A. Kirzhnits, Field Theoretical Methods in Many-Body Systems, Pergamon Press, London 1967.

28 I. S. Gradshteyn and I. M. Ryzhik, Tables of Integrals, Series, and Products, Academic Press, New York 1965.

29 Y. L. Luke, Integrals of Bessel Functions, McGraw-Hill, New York 1962.

30 E. T. Copson, Asymptotic Expansions, At The University Press, Cambridge 1965. 